\title{
What's in a Do-Not-Resuscitate Order? Understanding the Impact on Pre-arrest Life Support and Factors Influencing Misconceptions
}

\author{
Victoria T. Charoonratana, $B A^{7}$, Talia Stewart, $M H S^{7}{ }^{D}$, Runzhi Zhang, $M S^{2}$, \\ Zhigang Li, PhD'2, Martha T. DesBiens, MD, MPH ${ }^{1,3,4}$, Scott Slogic, BS, RRT ${ }^{5}$, and \\ Maxwell T. Vergo, $M D^{1,6}$
}

\begin{abstract}
'Section of Palliative Medicine, Geisel School of Medicine at Dartmouth Dartmouth-Hitchcock Medical Center, Lebanon, NH, USA; ${ }^{2}$ Department of Biostatistics, University of Florida, Gainsville, USA; ${ }^{3}$ Section of Infectious Disease, Dartmouth-Hitchcock Medical Center, Lebanon, USA; ${ }^{4}$ The Dartmouth Institute for Health Policy and Clinical Practice, Lebanon, USA; ${ }^{5}$ Life Safety, Dartmouth-Hitchcock Medical Center, Lebanon, USA: ${ }^{6}$ Section of Palliative Medicine, Dartmouth-Hitchcock Medical Center, Lebanon, USA.
\end{abstract}

Table 1 Demographics of Survey Respondents

\section{INTRODUCTION}

Discussion of code status in the event of cardiopulmonary arrest, such as FULL CODE or Do-Not-Resuscitate (DNR) status, has become the standard of care when a patient is admitted to the hospital. Some patients elect to avoid CPR, but have preferences for life support they are willing to receive prior to a cardiopulmonary arrest, which can be documented in an out-of-hospital Provider Orders for Life-Sustaining Treatments (POLST) form. ${ }^{1}$ The American Medical Association makes a clear statement that code status directs care at the time of cardiopulmonary arrest, and it should not impact life support delivered to a patient who is clinically deteriorating (still has a pulse and is breathing) unless otherwise directed by the patient. ${ }^{2}$

While studies have confirmed this association between DNR order status with care a provider would deliver when the patient still has a pulse and is breathing, ${ }^{3,4}$ few studies have examined how a provider's discipline, training background, and years of experience influence this bias with code status. The purpose of this study is to assess which health care team demographic factors are associated with misunderstanding of DNR order status.

\section{METHODS}

This institutional review board-approved study was a single timepoint, cross-sectional survey design of health care professionals at a 396-bed rural academic medical center in northern New England. Health care professionals were sent an institutional email with a voluntary and anonymous survey to collect demographic information, test factual knowledge of what a DNR order means,

Received August 20, 2019

Accepted September 24, 2019

Published online October 17, 2019

\begin{tabular}{|c|c|c|c|c|}
\hline Factors & & $\begin{array}{l}\text { Entire } \\
\text { sample } \\
N(\%)\end{array}$ & $\begin{array}{l}\text { Non- } \\
\text { critical } \\
\text { care } N \\
(\%)\end{array}$ & $\begin{array}{l}\text { Critical } \\
\text { care } N \\
(\%)\end{array}$ \\
\hline \multirow[t]{3}{*}{ Gender } & Female & $\begin{array}{l}186 \\
(69.4)\end{array}$ & $\begin{array}{l}108 \\
(94.7)\end{array}$ & $78(50.7)$ \\
\hline & Male & $77(28.7)$ & $5(4.4)$ & $72(46.8)$ \\
\hline & No response & $5(1.9)$ & $1(0.9)$ & $4(2.6)$ \\
\hline \multirow[t]{4}{*}{ Title } & $\begin{array}{l}\text { Graduate medical } \\
\text { education (intern, } \\
\text { resident, fellow) }\end{array}$ & $34(12.7)$ & 0 & $34(22.1)$ \\
\hline & $\begin{array}{l}\text { Attending } \\
\text { physician/ } \\
\text { advanced practice } \\
\text { provider (NP and } \\
\text { PA) }\end{array}$ & 37 (13.8) & $1(0.9)$ & $36(23.4)$ \\
\hline & Registered nurse & $\begin{array}{l}171 \\
(63.8)\end{array}$ & $\begin{array}{l}113 \\
(99.1)\end{array}$ & $58(37.7)$ \\
\hline & $\begin{array}{l}\text { Respiratory } \\
\text { therapist }\end{array}$ & $26(9.7)$ & 0 & $26(16.9)$ \\
\hline \multirow[t]{9}{*}{$\begin{array}{l}\text { Primary } \\
\text { specialty } \\
\text { training }\end{array}$} & $\begin{array}{l}\text { Internal medicine } \\
\text { and } \\
\text { subspecialties* }\end{array}$ & $43(16)$ & $9(7.9)$ & $34(21.9)$ \\
\hline & Cardiology & $19(7.1)$ & $12(10.5)$ & $7(4.5)$ \\
\hline & Neurology & 18 (6.7) & $7(6.1)$ & $11(7.1)$ \\
\hline & $\begin{array}{l}\text { Surgery (all } \\
\text { subspecialties } \\
\text { including cardiac) }\end{array}$ & $26(9.7)$ & $14(12.3)$ & $12(7.7)$ \\
\hline & Anesthesia & $13(4.9)$ & $1(0.9)$ & $12(7.7)$ \\
\hline & $\mathrm{PICU} / \mathrm{NICU}$ & $12(4.5)$ & $6(5.3)$ & $6(3.9)$ \\
\hline & $\begin{array}{l}\text { Nursing }(\mathrm{RN}) / \text { ad- } \\
\text { vanced practice } \\
\text { provider (NP and } \\
\text { PA) }\end{array}$ & $94(35.1)$ & $65(57)$ & $29(18.7)$ \\
\hline & $\begin{array}{l}\text { Emergency } \\
\text { medicine }\end{array}$ & $17(6.3)$ & 0 & $17(11)$ \\
\hline & $\begin{array}{l}\text { Respiratory } \\
\text { therapy }\end{array}$ & $26(9.7)$ & 0 & $26(16.8)$ \\
\hline \multirow{5}{*}{$\begin{array}{l}\text { Level of } \\
\text { experience }\end{array}$} & Current resident & $27(10.1)$ & 0 & $27(17.5)$ \\
\hline & $0-5$ years & & $58(50.9)$ & $43(27.9)$ \\
\hline & $6-10$ years & $45(16.8)$ & $20(17.5)$ & $25(16.2)$ \\
\hline & $>10$ years & $89(33.1)$ & $33(29)$ & $56(36.4)$ \\
\hline & No response & $6(2.2)$ & $3(2.6)$ & $3(2.0)$ \\
\hline
\end{tabular}

PICU pediatric intensive care unit, NICU neonatal intensive care unit, $R N$ registered nurse, NP nurse practitioner, PA physician assistant *The "Internal medicine and subspecialties" category includes hospitalists, hematology/oncology, pulmonology, nephrology, and palliative care 
and apply this knowledge to clinical vignettes. The vignettes assessed the use of life-sustaining treatments (LSTs) in clinically deteriorating patients with a DNR order, including transfer to the intensive care unit (ICU), antibiotics, vasopressors, transfusions, dialysis, synchronized cardioversion, non-invasive positive pressure ventilation, and intubation.

To assess the internal validity of the survey, we used the KR-20 coefficient. We used the Pearson chi-square test (or Fisher's exact test if necessary) to assess raw associations of factors with incorrect interpretation of DNR orders. We used stepwise selection to choose the final logistic regression model for the binary interpretation of DNR orders to include covariates with a $p$ value of 0.1 or less in the final model. All statistical analyses were performed using $\mathrm{R}$ version 3.5.2.

\section{RESULTS}

A total of 268 completed the survey $(n=268)$ out of 321 surveys sent (response rate $=83 \%$ ). Baseline demographics of responding health care professionals are included in Table 1. Most survey participants incorrectly defined DNR order status $(n=236,88 \%)$ and incorrectly avoided other LSTs prior to a cardiopulmonary arrest based on DNR order status in the clinical vignettes $(n=232,87 \%)$. These questions were significantly and positively correlated $(p<0.0001)$ based on Fisher-exact test with a KR-20 coefficient of 0.903 , meaning that participants either answered both questions correctly or incorrectly in a consistent fashion.

We examined the association between incorrectly defining DNR order status and the decision to limit other LSTs prior to

Table 2 Statistical Analysis of Factors Associated with Misunderstanding of Do-Not-Resuscitate Status

\begin{tabular}{|c|c|c|c|c|c|c|c|}
\hline Factors & & $\begin{array}{l}\text { Raw } \\
\text { association }\end{array}$ & $p$ value & $\begin{array}{l}\text { Full model } \\
\text { (univariate) }\end{array}$ & $p$ value & $\begin{array}{l}\text { Final Model } \\
\text { (Multivariate) }\end{array}$ & p Value \\
\hline \multirow[t]{2}{*}{ Work environment } & Non-critical care & \multicolumn{6}{|l|}{ Reference group } \\
\hline & Critical care & $\begin{array}{l}\text { OR } 1.87 \\
(0.82-4.26)\end{array}$ & $0.190(\mathrm{NS})$ & -0.32 & $0.65(\mathrm{NS})$ & - & - \\
\hline \multirow[t]{4}{*}{ Job title } & $\begin{array}{l}\text { Graduate medical } \\
\text { education (intern, } \\
\text { resident, fellow) }\end{array}$ & \multicolumn{6}{|l|}{ Reference group } \\
\hline & $\begin{array}{l}\text { Attending physician/ } \\
\text { advanced practice pro- } \\
\text { vider (NP and PA) }\end{array}$ & -0.76 & 0.28 (NS) & - & - & - & - \\
\hline & Registered nurse & -1.15 & $0.02585^{*}$ & - & - & - & - \\
\hline & Respiratory therapist & & 0.74 (NS) & - & - & - & - \\
\hline \multirow[t]{2}{*}{ Gender } & Female & \multicolumn{6}{|c|}{ Reference group } \\
\hline & Male & $\begin{array}{l}\text { OR } 1.27 \\
(0.57-2.87)\end{array}$ & 0.71 (NS) & -0.71 & $0.26(\mathrm{NS})$ & - & - \\
\hline \multirow[t]{9}{*}{ Primary specialty } & $\begin{array}{l}\text { Internal medicine and } \\
\text { subspecialties }\end{array}$ & \multicolumn{6}{|l|}{ Reference group } \\
\hline & Cardiology & -1.57 & 0.15 (NS) & -1.21 & $0.35(\mathrm{NS})$ & - & - \\
\hline & Neurology & -16.50 & $1.0(\mathrm{NS})$ & -18.42 & 0.99 (NS) & - & - \\
\hline & $\begin{array}{l}\text { Surgery (all } \\
\text { subspecialties } \\
\text { including cardiac) }\end{array}$ & -2.15 & $0.04601 *$ & -2.88 & $0.027889 *$ & - & - \\
\hline & Anesthesia & -1.12 & $0.31(\mathrm{NS})$ & -0.33 & $0.81(\mathrm{NS})$ & - & - \\
\hline & PICU/NICU & -1.33 & $0.23(\mathrm{NS})$ & -1.06 & $0.42(\mathrm{NS})$ & - & - \\
\hline & $\begin{array}{l}\text { Nursing }(\mathrm{RN}) / \text { ad- } \\
\text { vanced practice pro- } \\
\text { vider (NP and PA) }\end{array}$ & -1.59 & $0.00362 *$ & -1.84 & $0.025874 *$ & - & - \\
\hline & Emergency medicine & -0.48 & $0.52(\mathrm{NS})$ & -0.33 & $0.74(\mathrm{NS})$ & - & - \\
\hline & Respiratory therapy & -0.03 & $0.96(\mathrm{NS})$ & -0.87 & 0.29 (NS) & - & - \\
\hline \multirow{9}{*}{$\begin{array}{l}\text { Level of experience } \\
\text { Confusion over use of a } \\
\text { specific medical } \\
\text { intervention in patients } \\
\text { with DNR order status }\end{array}$} & & -0.37 & 0.057 (NS) & -0.30 & $0.27(\mathrm{NS})$ & - & - \\
\hline & $\begin{array}{l}\text { DNR order status } \\
\text { influencing ICU } \\
\text { transfer }\end{array}$ & $\begin{array}{l}\text { OR } 1.69 \\
(0.48-9.18)\end{array}$ & 0.59 (NS) & -1.26 & $0.14(\mathrm{NS})$ & - & - \\
\hline & $\begin{array}{l}\text { DNR order status } \\
\text { influencing antibiotics }\end{array}$ & $\begin{array}{l}\text { Fisher's exact } \\
\text { test }\end{array}$ & 0.60 (NS) & 13.07 & 1.0 (NS) & - & - \\
\hline & $\begin{array}{l}\text { DNR order status } \\
\text { influencing } \\
\text { vasopressors }\end{array}$ & $\begin{array}{l}\text { OR 5.96 } \\
(1.43-53.02)\end{array}$ & $0.007526^{*}$ & 0.59 & $0.54(\mathrm{NS})$ & - & - \\
\hline & $\begin{array}{l}\text { DNR order status } \\
\text { influencing transfusion }\end{array}$ & $\begin{array}{l}\text { Fisher's exact } \\
\text { test }\end{array}$ & $\begin{array}{l}0.08689 \\
(\mathrm{NS})\end{array}$ & 16.67 & 0.99 (NS) & 15.83 & $\begin{array}{l}0.99 \\
\text { (NS) }\end{array}$ \\
\hline & $\begin{array}{l}\text { DNR order status } \\
\text { influencing dialysis }\end{array}$ & $\begin{array}{l}\text { OR } 1.56 \\
(0.57-4.27)\end{array}$ & $0.52(\mathrm{NS})$ & -1.04 & 0.16 (NS) & - & - \\
\hline & $\begin{array}{l}\text { DNR order status } \\
\text { influencing } \\
\text { cardioversion }\end{array}$ & $\begin{array}{l}\text { OR } 3.75 \\
(1.60-8.79)\end{array}$ & $0.002598^{*}$ & 0.50 & $0.40(\mathrm{NS})$ & - & - \\
\hline & $\begin{array}{l}\text { DNR order status } \\
\text { influencing BiPap } \\
\text { administration }\end{array}$ & $\begin{array}{l}\text { OR } 14.51 \\
(2.31- \\
602.27)\end{array}$ & $<0.001 *$ & 1.42 & 0.24 (NS) & 1.66 & $\begin{array}{l}0.12 \\
\text { (NS) }\end{array}$ \\
\hline & $\begin{array}{l}\text { DNR order status } \\
\text { influencing intubation }\end{array}$ & $\begin{array}{l}\text { OR 10.28 } \\
(4.39-24.04)\end{array}$ & $<0.001 *$ & 2.18 & $<0.001 *$ & 1.93 & $<0.001 *$ \\
\hline
\end{tabular}

NS no statistical significance, DNR do-not-resuscitate, PICU pediatric intensive care unit, NICU neonatal intensive care unit, RN registered nurse, NP nurse practitioner, PA physician assistant, BiPAP bilevel positive airway pressure, ICU intensive care unit

*Indicates statistical significance 
a cardiopulmonary arrest. Table 2 includes the univariate and multivariate analyses. In a multivariate analysis, only limitation for intubation remained significantly associated with clinicians who incorrectly defined DNR order status. In addition, we examined the association between incorrectly defining DNR order status and other clinician factors. The multivariate analysis only remained significant for the specialties of nursing/advance practice provider and surgery. Of note, there was no association between incorrect definition of DNR order status and gender, level of experience, clinical environment, limiting antibiotics, transfusions, dialysis, or intensive care unit admission.

\section{DISCUSSION}

In summary, our study demonstrated that all providers are at risk for misinterpreting DNR orders in our current system. Our results suggest that nurses and surgical subspecialties are at highest risk for limiting life support, particularly mechanical ventilation, in a patient with a DNR order prior to cardiopulmonary arrest. Our results can inform educational efforts on code status, directing education about the interventions most likely to cause confusion towards the providers at highest risk for misinterpretation. This is consistent with previous studies that have shown higher rates of DNR misinterpretation among nurses and an increased likelihood of limiting general surgery procedures in patients with DNR orders. ${ }^{5,6}$ To address this issue, hospital systems should consider designing systems that separate code status from orders for pre-arrest care, following the distinction seen in POLST forms.

Acknowledgments: The authors thank Dr. Alan Garber and Dr. Jeffrey Munson for substantial help in creating this survey.
Corresponding Author: Talia Stewart, MHS; Section of Palliative MedicineGeisel School of Medicine at Dartmouth Dartmouth-Hitchcock Medical Center, Lebanon, NH 03756, USA (e-mail: talia.f.stewart. med@dartmouth.edu).

\section{Compliance with Ethical Standards:}

Conflict of Interest: The authors declare that they do not have a conflict of interest.

\section{REFERENCES}

1. Mirarchi FL, Cooney TE, Venkat A, et al. TRIAD VIII: Nationwide multicenter evaluation to determine whether patient video testimonials can safely help ensure appropriate critical versus end-of-life care. J Patient Saf. 2017. doi:https://doi.org/10.1097/PTS.0000000000000357

2. Wik L, Kramer-Johansen J, Myklebust H, et al. Quality of cardiopulmonary resuscitation during out-of-hospital cardiac arrest. J Am Med Assoc. 2005. doi:https://doi.org/10.1001/jama.293.3.299

3. Puma J, Silverstein MD, Stocking CB, et al. Life-Sustaining Treatment: A Prospective Study of Patients With DNR Orders in a Teaching Hospital. Arch Intern Med. 1988;148(10):2193-2198. doi:https://doi.org/10.1001/ archinte.1988.00380100067015

4. Stevenson EK, Mehter HM, Walkey AJ, et al. Association between do not resuscitate/do not intubate status and resident physician decisionmaking a national survey. Ann Am Thorac Soc. 2017;14(4):536-542. doi:https://doi.org/10.1513/AnnalsATS.201610-798OC

5. Scarborough JE, Pappas TN, Bennett KM, Lagoo-Deenadayalan S. Failureto-pursue rescue: Explaining excess mortality in elderly emergency general surgical patients with preexisting do-not-resuscitate orders. In: Annals of Surgery. ; 2012. doi:https://doi.org/10.1097/SLA.0b013e31826578fb

6. O’Brien H, Scarlett S, Brady A, Harkin K, Kenny RA, Moriarty J. Do-notattempt-resuscitation (DNAR) orders: Understanding and interpretation of their use in the hospitalised patient in Ireland. A brief report. J Med Ethics. 2018;44(3):201-203. doi:https://doi.org/10.1136/medethics-2016103986

Publisher's Note: Springer Nature remains neutral with regard to jurisdictional claims in published maps and institutional affiliations. 